\title{
Ammonium boranes for the selective complexation of cyanide or fluoride ions in water
}

\section{Supplementary Information}

Todd W. Hudnall and François P. Gabbai"**

$\dagger$ Department of Chemistry, Texas A\&M University, College Station, Texas 77843 
Complete reference 66: Yuan, Z.; Entwistle, C. D.; Collings, J. C.; Albesa-Jove, D.; Batsanov, A. S.; Howard, J. A. K.; Taylor, N. J.; Kaiser, H. M.; Kaufmann, D. E.; Poon, S.-Y.; Wong, W.Y.; Jardin, C.; Fathallah, S.; Boucekkine, A.; Halet, J.-F.; Marder, T. B. Chem. Eur. J. 2006, 12, 2758.

\section{UV-vis titration experiments}

All titration experiments were carried out in $\mathrm{H}_{2} \mathrm{O} /$ DMSO 6:4 (HEPES $6 \mathrm{mM}$ in $\mathrm{H}_{2} \mathrm{O}$, pH 7). We verified that the $\mathrm{pH}$ of this mixture was 7 using a SympHony Gel-Filled Combination Electrode from VWR International. The $\mathrm{pK}_{\mathrm{a}}$ of $\mathrm{HCN}$ was also measured (potentiometric titration of $\mathrm{NaCN}$ with $\mathrm{HCl})$ in $\mathrm{H}_{2} \mathrm{O} / \mathrm{DMSO} 6 / 4$ and found to be equal $9.3 \pm(0.01)$.

\section{Titration of $[\mathbf{1}]^{+}$in $\mathrm{H}_{2} \mathrm{O} / \mathrm{DMSO}$}

A solution of [1]OTf $\left(3.0 \mathrm{~mL}, 4.8739 \times 10^{-5} \mathrm{M}\right.$, in buffered $\mathrm{H}_{2} \mathrm{O} / \mathrm{DMSO} 6 / 4$ vol. (HEPES $6 \mathrm{mM}$ in $\mathrm{H}_{2} \mathrm{O}$, pH 7) was titrated with incremental amounts of cyanide anions by addition of a solution of $\mathrm{NaCN}$ in $\mathrm{H}_{2} \mathrm{O}\left(3.0 \times 10^{-3} \mathrm{M}\right)$. The absorption was monitored at $\lambda_{\max }=320 \mathrm{~nm}(\varepsilon=8376)$. The experimental data obtained was fitted by hand to a $1: 1$ binding isotherm using the equation provided below. This equation was derived in order to account for the competitive protonation of the cyanide ion (See Table S1, Figure S1).

Derivation of the cyanide titration isotherm

\begin{tabular}{|c|c|c|c|c|c|c|c|c|}
\hline $1^{+}$ & + & $\mathrm{H}^{+}$ & + & $\mathrm{NaCN}$ & $\Leftrightarrow$ & 1-CN & + & $\mathrm{HCN}$ \\
\hline $\mathbf{1}_{0}^{+}-\mathrm{x}$ & & $\mathrm{H}^{+}$ & & {$[\mathrm{NaCN}]_{0}-\mathrm{x}-\mathrm{y}$} & & $\mathrm{x}$ & & $\mathrm{y}$ \\
\hline
\end{tabular}

$\mathrm{K}_{[\mathbf{1}+]}=[\mathbf{1}-\mathrm{CN}] /\left(\left[\mathbf{1}^{+}\right]^{*}[\mathrm{NaCN}]\right)=\mathrm{x} /\left[\left(\left[\mathbf{1}^{+}\right]_{0}-\mathrm{x}\right) *\left([\mathrm{NaCN}]_{0}-\mathrm{x}-\mathrm{y}\right)\right]$

$\mathrm{K}_{2}=[\mathrm{HCN}] /\left(\left[\mathrm{H}^{+}\right] *[\mathrm{NaCN}]\right)=\mathrm{y} /\left[\left(\mathrm{H}^{+}\right) *\left([\mathrm{NaCN}]_{0}-\mathrm{x}-\mathrm{y}\right)\right]=1 / \mathrm{K}_{\mathrm{a}}$ of $\mathrm{HCN}$

$\left.\Rightarrow \mathrm{K}_{\mathrm{rel}}=\mathrm{K}_{[\mathbf{1 +}]} / \mathrm{K}_{2}=\left[\mathrm{x} *\left[\mathrm{H}^{+}\right]\right)\right] /\left[\mathrm{y} *\left(\left[\mathbf{1}^{+}\right]_{0}-\mathrm{x}\right)\right]$

$\mathrm{K}_{[1+]}$ and $\mathrm{K}_{2}$ are very large, and therefore there is very little free cyanide anion.

As a result, the following approximation can be made: $[\mathrm{NaCN}]=\mathrm{x}+\mathrm{y}$

Therefore $\mathrm{y}=[\mathrm{NaCN}]-\mathrm{x}$

$\Rightarrow \mathrm{K}_{\mathrm{rel}}=\left[\mathrm{x} *\left[\mathrm{H}^{+}\right]\right] /\left[([\mathrm{NaCN}]-\mathrm{x}) *\left(\left[\mathbf{1}^{+}\right]_{0}-\mathrm{x}\right)\right]$

$\Rightarrow([\mathrm{NaCN}]-\mathrm{x})^{*}\left(\left[\mathbf{1}^{+}\right]_{0}-\mathrm{x}\right)=\mathrm{x} *\left(\left[\mathrm{H}^{+}\right] / \mathrm{K}_{\mathrm{rel}}\right)=[\mathrm{NaCN}]^{*}\left[\mathbf{1}^{+}\right]_{0}+\mathrm{x}^{2}-\mathrm{x}^{*}\left([\mathrm{NaCN}]+\left[\mathbf{1}_{0}^{+}\right]\right.$ 


$$
\begin{aligned}
& \Rightarrow \mathrm{x}^{2}-\mathrm{x} *\left([\mathrm{NaCN}]+\left[\mathbf{1}^{+}\right]_{0}\right)-\mathrm{x}^{*}\left(\left[\mathrm{H}^{+}\right] / \mathrm{K}_{\mathrm{rel}}\right)+\left[\mathbf{1}^{+}\right]_{0} *[\mathrm{NaCN}]=0 \\
& =\underbrace{2}_{\mathrm{a}}-\mathrm{x}^{*}\left([\mathrm{NaCN}]+\left[\mathbf{1}^{+}\right]_{0}+\left(\left[\mathrm{H}^{+}\right] / \mathrm{K}_{\mathrm{rel}}\right)\right)+\mathbf{1}^{+}]_{0} *[\mathrm{NaCN}])=0 \\
& =>\mathrm{x}=\left[-\mathrm{b}-(\Delta)^{1 / 2}\right] / 2 \mathrm{a} \text { with } \Delta=\mathrm{b}^{2}-4 \mathrm{ac} \\
& =>\mathrm{Abs}_{\text {calc }}=\left(\left[\mathbf{1}^{+}\right]_{0}-\mathrm{x}\right) * \varepsilon
\end{aligned}
$$

The experimental data obtained was fitted by hand using the equation above. This fit yielded a $\mathrm{K}_{\mathrm{rel}}=2.00( \pm 0.5) \times 10^{-1} \mathrm{M}^{-1}$. Since $\mathrm{K}_{\mathrm{a}}$ for $\mathrm{HCN}=5.01 \times 10^{-10} \mathrm{M}^{-1} \Rightarrow$ the stability constant of 1$\mathrm{CN}, \mathrm{K}_{[1+]}$ is equal to $3.9 \times 10^{8} \mathrm{M}^{-1}$.

Table S1. Absorbance of a solution of $[1]^{+}$after successive additions of cyanide in buffered $\mathrm{H}_{2} \mathrm{O} / \mathrm{DMSO} 6 / 4$ vol. (HEPES 0.01 $\mathrm{M}$ in $\mathrm{H}_{2} \mathrm{O}, \mathrm{pH}$ 7)

\begin{tabular}{lll|lcc}
\hline $\mathrm{C}_{\text {Fluoride }}$ & $\mathrm{Abs}_{\text {exp }}$ & $\mathrm{Abs}_{\text {calc }}$ & $\mathrm{C}_{\text {Fluoride }}$ & $\mathrm{Abs}_{\text {exp }}$ & Abs $_{\text {calc }}$ \\
\hline $0.0000 \mathrm{E} 00$ & $4.08 \mathrm{E}-01$ & $4.08 \mathrm{E}-01$ & $4.8213 \mathrm{E}-05$ & $4.47 \mathrm{E}-02$ & $3.80 \mathrm{E}-02$ \\
$6.9837 \mathrm{E}-06$ & $3.67 \mathrm{E}-01$ & $3.49 \mathrm{E}-01$ & $5.4974 \mathrm{E}-05$ & $1.93 \mathrm{E}-02$ & $2.00 \mathrm{E}-02$ \\
$1.3935 \mathrm{E}-05$ & $3.06 \mathrm{E}-01$ & $2.91 \mathrm{E}-01$ & $6.1704 \mathrm{E}-05$ & $9.62 \mathrm{E}-03$ & $1.25 \mathrm{E}-02$ \\
$2.0854 \mathrm{E}-05$ & $2.39 \mathrm{E}-01$ & $2.34 \mathrm{E}-01$ & $6.8404 \mathrm{E}-05$ & $7.64 \mathrm{E}-03$ & $8.93 \mathrm{E}-03$ \\
$2.7741 \mathrm{E}-05$ & $1.86 \mathrm{E}-01$ & $1.77 \mathrm{E}-01$ & $7.5073 \mathrm{E}-05$ & $5.17 \mathrm{E}-03$ & $6.89 \mathrm{E}-03$ \\
$3.4596 \mathrm{E}-05$ & $1.32 \mathrm{E}-01$ & $1.23 \mathrm{E}-01$ & $8.1712 \mathrm{E}-05$ & $4.38 \mathrm{E}-03$ & $5.60 \mathrm{E}-03$ \\
$4.1420 \mathrm{E}-05$ & $8.33 \mathrm{E}-02$ & $7.42 \mathrm{E}-02$ & --- & --- & --
\end{tabular}

Titration of $[2]^{+}$in $\mathrm{H}_{2} \underline{2}$ O/DMSO

A solution of [2]OTf $\left(3.0 \mathrm{~mL}, 5.1500 \times 10^{-5} \mathrm{M}\right.$, in buffered $\mathrm{H}_{2} \mathrm{O} / \mathrm{DMSO} 6 / 4$ vol. (HEPES $6 \mathrm{mM}$, $\mathrm{pH} 7$ ) was titrated with incremental amounts of fluoride anions by addition of a solution of $\mathrm{NaF}$ in $\mathrm{H}_{2} \mathrm{O}\left(3.01 \times 10^{-1} \mathrm{M}\right)$. The absorption was monitored at $\lambda_{\max }=320 \mathrm{~nm}(\varepsilon=9200)$. The experimental data obtained was fitted by hand to a 1:1 binding isotherm to afford a stability constant for $2-\mathrm{F}$ of $910 \pm(50) \mathrm{M}^{-1}$. (See Table S2).

Table S2. Absorbance of a solution of [2] ${ }^{+}$after successive additions of fluoride in buffered $\mathrm{H}_{2} \mathrm{O} / \mathrm{DMSO} 6 / 4$ vol. (HEPES 0.01 $\mathrm{M}$ in $\mathrm{H}_{2} \mathrm{O}$, pH 7)

\begin{tabular}{lll}
\hline $\mathrm{C}_{\text {Fluoride }}$ & $\mathrm{Abs}_{\exp }$ & $\mathrm{Abs}_{\text {calc }}$ \\
\hline $0.00 \mathrm{E}+00$ & $4.79 \mathrm{E}-01$ & $4.74 \mathrm{E}-01$ \\
$4.01 \mathrm{E}-04$ & $3.58 \mathrm{E}-01$ & $3.50 \mathrm{E}-01$ \\
$8.01 \mathrm{E}-04$ & $2.74 \mathrm{E}-01$ & $2.76 \mathrm{E}-01$
\end{tabular}




\section{Optimized structure of $[\mathbf{1}]^{+}$}

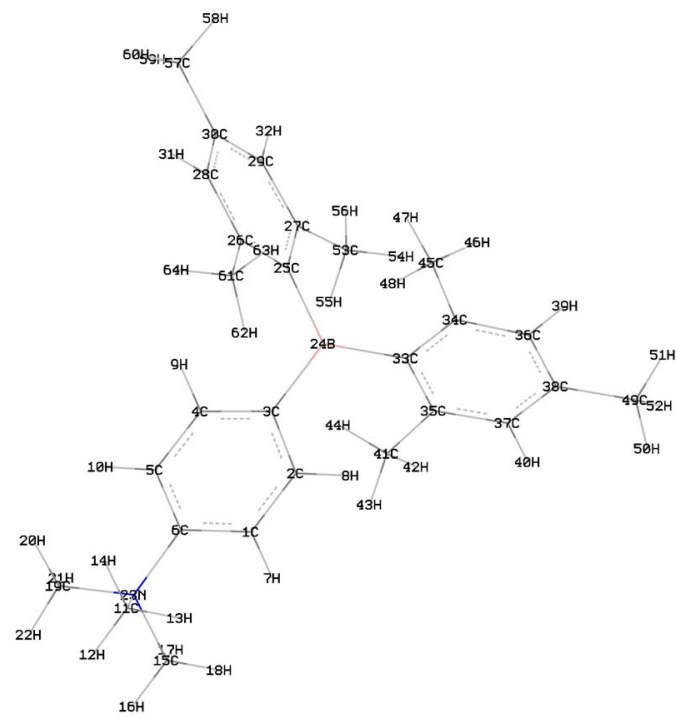

\begin{tabular}{|c|c|c|c|c|}
\hline$\#$ & Symbol & $X$ & $Y$ & $Z$ \\
\hline 1 & C & -2.992615 & -1.030419 & 0.613240 \\
\hline 2 & C & -1.599893 & -1.033285 & 0.609142 \\
\hline 3 & C & -0.851862 & 0.004703 & 0.019738 \\
\hline 4 & C & -1.578734 & 1.046704 & -0.579991 \\
\hline 5 & C & -2.976115 & 1.062829 & -0.611985 \\
\hline 6 & C & -3.675898 & 0.020701 & -0.006308 \\
\hline 7 & H & -3.518658 & -1.850994 & 1.091250 \\
\hline 8 & H & -1.077338 & -1.863079 & 1.076122 \\
\hline 9 & H & -1.041556 & 1.873189 & -1.035337 \\
\hline 10 & H & -3.473655 & 1.890438 & -1.100720 \\
\hline 11 & C & -5.697302 & -0.020504 & 1.439491 \\
\hline 12 & H & -6.788322 & -0.031690 & 1.426317 \\
\hline 13 & H & -5.321150 & -0.904041 & 1.950927 \\
\hline 14 & H & -5.329484 & 0.877046 & 1.936733 \\
\hline 15 & C & -5.678571 & -1.266196 & -0.717626 \\
\hline 16 & H & -6.769739 & -1.271741 & -0.711604 \\
\hline 17 & H & -5.301735 & -1.242546 & -1.740211 \\
\hline 18 & H & -5.297627 & -2.147310 & -0.205707 \\
\hline 19 & C & -5.810298 & 1.184591 & -0.695757 \\
\hline 20 & H & -5.503357 & 2.097387 & -0.186949 \\
\hline 21 & H & -5.490681 & 1.201831 & -1.736791 \\
\hline 22 & H & -6.894100 & 1.078729 & -0.643872 \\
\hline 23 & N & -5.191073 & -0.012967 & 0.001159 \\
\hline 24 & B & 0.746013 & -0.001570 & 0.028439 \\
\hline 25 & C & 1.474353 & 1.390650 & -0.018499 \\
\hline 26 & C & 1.208296 & 2.420171 & 0.923860 \\
\hline 27 & C & 2.392292 & 1.676562 & -1.074179 \\
\hline 28 & C & 1.815248 & 3.675131 & 0.790913 \\
\hline 29 & C & 2.960875 & 2.946836 & -1.177436 \\
\hline 30 & C & 2.691086 & 3.964966 & -0.254457 \\
\hline 31 & H & 1.604108 & 4.442314 & 1.534068 \\
\hline 32 & H & 3.638940 & 3.150815 & -2.003920 \\
\hline & & & & \\
\hline
\end{tabular}

\begin{tabular}{|l|l|l|l|l|}
\hline 33 & C & 1.474507 & -1.393181 & 0.056830 \\
\hline 34 & C & 2.464288 & -1.665017 & 1.047632 \\
\hline 35 & C & 1.162675 & -2.424902 & -0.870143 \\
\hline 36 & C & 3.067847 & -2.922882 & 1.104058 \\
\hline 37 & C & 1.817943 & -3.658743 & -0.797674 \\
\hline 38 & C & 2.767745 & -3.936027 & 0.186950 \\
\hline 39 & H & 3.804733 & -3.116176 & 1.881156 \\
\hline 40 & H & 1.584005 & -4.423292 & -1.536469 \\
\hline 41 & C & 0.159868 & -2.244275 & -2.000305 \\
\hline 42 & H & 0.444063 & -2.858409 & -2.861385 \\
\hline 43 & H & -0.850073 & -2.556401 & -1.702844 \\
\hline 44 & H & 0.088990 & -1.209309 & -2.347203 \\
\hline 45 & C & 2.877901 & -0.637229 & 2.085740 \\
\hline 46 & H & 3.602249 & -1.067740 & 2.783801 \\
\hline 47 & H & 3.339628 & 0.241767 & 1.624580 \\
\hline 48 & H & 2.027717 & -0.279374 & 2.678982 \\
\hline 49 & C & 3.471203 & -5.271940 & 0.247351 \\
\hline 50 & H & 2.979288 & -6.014780 & -0.388336 \\
\hline 51 & H & 4.512385 & -5.185720 & -0.090134 \\
\hline 52 & H & 3.498337 & -5.664161 & 1.270569 \\
\hline 53 & C & 2.758241 & 0.651568 & -2.132854 \\
\hline 54 & H & 3.232727 & -0.232816 & -1.696328 \\
\hline 55 & H & 1.883695 & 0.300760 & -2.695056 \\
\hline 56 & H & 3.455687 & 1.082215 & -2.857711 \\
\hline 57 & C & 3.347526 & 5.320198 & -0.380446 \\
\hline 58 & H & 4.415452 & 5.266361 & -0.131644 \\
\hline 59 & H & 3.277115 & 5.706160 & -1.404109 \\
\hline 60 & H & 2.889055 & 6.052922 & 0.290977 \\
\hline 61 & C & 0.368101 & 2.194189 & 2.168415 \\
\hline 62 & H & -0.320038 & 1.350440 & 2.076646 \\
\hline 63 & H & 1.013875 & 1.988953 & 3.033111 \\
\hline 64 & H & -0.219339 & 3.085701 & 2.416586 \\
\hline & & & & \\
\hline 50
\end{tabular}




\section{Optimized structure of $[2]^{+}$}

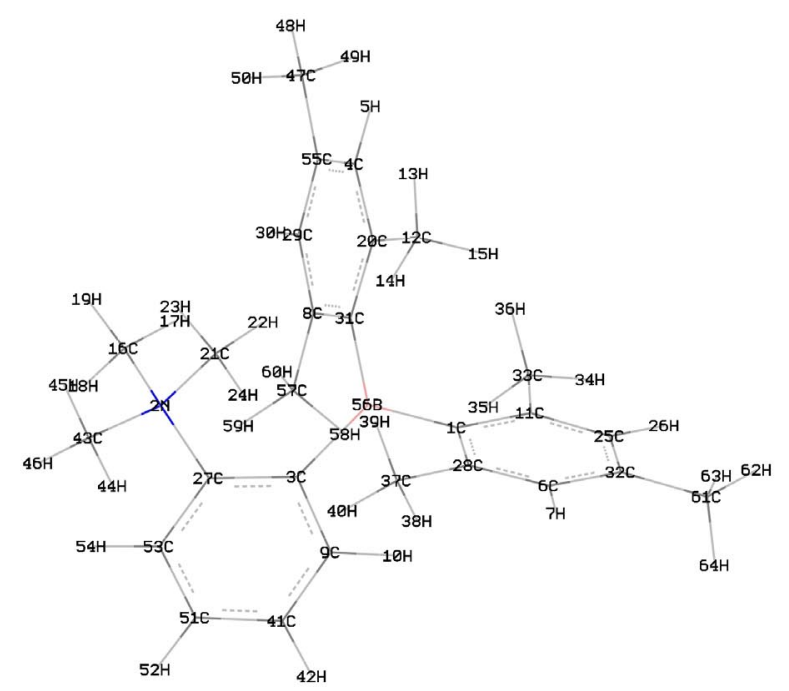

\begin{tabular}{|c|c|c|c|c|}
\hline \# & Symbol & $x$ & $Y$ & $z$ \\
\hline 1 & C & 1.847447 & 0.015348 & -0.003557 \\
\hline 2 & $N$ & -2.391828 & -1.754511 & -1.153808 \\
\hline 3 & C & -0.361242 & -1.516848 & 0.445975 \\
\hline 4 & C & -1.345275 & 3.303891 & -0.923919 \\
\hline 5 & H & -1.245986 & 4.017002 & -1.739737 \\
\hline 6 & C & 3.994425 & -0.733525 & -0.922900 \\
\hline 7 & $\mathrm{H}$ & 4.541907 & -1.386398 & -1.599715 \\
\hline 8 & C & -1.589796 & 1.465239 & 1.163744 \\
\hline 9 & C & 0.336846 & -2.131702 & 1.515651 \\
\hline 10 & $\mathrm{H}$ & 1.275767 & -1.684329 & 1.823937 \\
\hline 11 & C & 2.573986 & 0.963261 & 0.781792 \\
\hline 12 & C & 0.529946 & 2.081449 & -2.012604 \\
\hline 13 & $\mathrm{H}$ & 0.290083 & 2.775990 & -2.823480 \\
\hline 14 & $\mathrm{H}$ & 0.608837 & 1.079605 & -2.448449 \\
\hline 15 & $\mathrm{H}$ & 1.530952 & 2.334753 & -1.646355 \\
\hline 16 & C & .3 .538506 & -0.869348 & -0.680212 \\
\hline 17 & $\mathrm{H}$ & -3.120970 & 0.031517 & -0.232254 \\
\hline 18 & $\mathrm{H}$ & -4.121184 & -1.422969 & 0.055842 \\
\hline 19 & $\mathrm{H}$ & -4.159784 & -0.613484 & -1.540952 \\
\hline 20 & C & -0.502849 & 2.186332 & -0.901084 \\
\hline 21 & C & -1.586335 & -0.995531 & -2.191146 \\
\hline 22 & H & -1.247484 & -0.059291 & -1.761242 \\
\hline 23 & $\mathrm{H}$ & -2.240313 & -0.785820 & -3.038648 \\
\hline 24 & $\mathrm{H}$ & -0.747114 & -1.612963 & -2.504868 \\
\hline 25 & C & 3.969561 & 1.014970 & 0.703542 \\
\hline 26 & $\mathrm{H}$ & 4.500525 & 1.729384 & 1.328712 \\
\hline 27 & C & -1.542029 & -2.190110 & 0.047799 \\
\hline 28 & C & 2.604521 & -0.842857 & -0.859403 \\
\hline 29 & C & -2.384196 & 2.615469 & 1.115475 \\
\hline 30 & $\mathrm{H}$ & -3.090866 & 2.797880 & 1.922670 \\
\hline 31 & C & -0.632010 & 1.213534 & 0.135100 \\
\hline 32 & C & 4.704689 & 0.185101 & -0.144654 \\
\hline
\end{tabular}

\begin{tabular}{|l|l|l|l|l|}
\hline 33 & C & 1.914901 & 1.922218 & 1.759510 \\
\hline 34 & H & 2.676214 & 2.427939 & 2.360707 \\
\hline 35 & H & 1.237648 & 1.416564 & 2.456208 \\
\hline 36 & H & 1.331012 & 2.694663 & 1.249180 \\
\hline 37 & C & 1.962788 & -1.847388 & -1.799373 \\
\hline 38 & H & 2.716083 & -2.529952 & -2.203755 \\
\hline 39 & H & 1.496872 & -1.348223 & -2.661328 \\
\hline 40 & H & 1.199695 & -2.455632 & -1.305735 \\
\hline 41 & C & -0.123518 & -3.251222 & 2.203883 \\
\hline 42 & H & 0.455657 & -3.660574 & 3.025885 \\
\hline 43 & C & -2.981514 & -2.956074 & -1.886940 \\
\hline 44 & H & -2.183848 & -3.668444 & -2.095852 \\
\hline 45 & H & -3.421264 & -2.596222 & -2.817259 \\
\hline 46 & H & -3.759731 & -3.422934 & -1.289292 \\
\hline 47 & C & -3.150461 & 4.787720 & 0.056867 \\
\hline 48 & H & -3.323884 & 5.142439 & -0.964157 \\
\hline 49 & H & -2.666812 & 5.606114 & 0.606592 \\
\hline 50 & H & -4.122627 & 4.612462 & 0.529192 \\
\hline 51 & C & -1.327466 & -3.832345 & 1.824863 \\
\hline 52 & H & -1.723446 & -4.698279 & 2.345747 \\
\hline 53 & C & -2.023105 & -3.307580 & 0.736487 \\
\hline 54 & H & -2.941862 & -3.800043 & 0.446183 \\
\hline 55 & C & -2.290367 & 3.546283 & 0.075416 \\
\hline 56 & B & 0.284577 & -0.075693 & 0.127477 \\
\hline 57 & C & -1.750006 & 0.568419 & 2.382112 \\
\hline 58 & H & -0.790158 & 0.344308 & 2.859393 \\
\hline 59 & H & -2.216587 & -0.395587 & 2.149013 \\
\hline 60 & H & -2.377252 & 1.059371 & 3.132057 \\
\hline 61 & C & 6.211424 & 0.258582 & -0.207258 \\
\hline 62 & H & 6.591029 & 1.158112 & 0.286319 \\
\hline 63 & H & 6.568769 & 0.261924 & -1.243292 \\
\hline 64 & H & 6.668882 & -0.608563 & 0.287007 \\
\hline & & & & \\
\hline 5
\end{tabular}




\section{Optimized structure of $\mathrm{Mes}_{2} \mathrm{BPh}$}

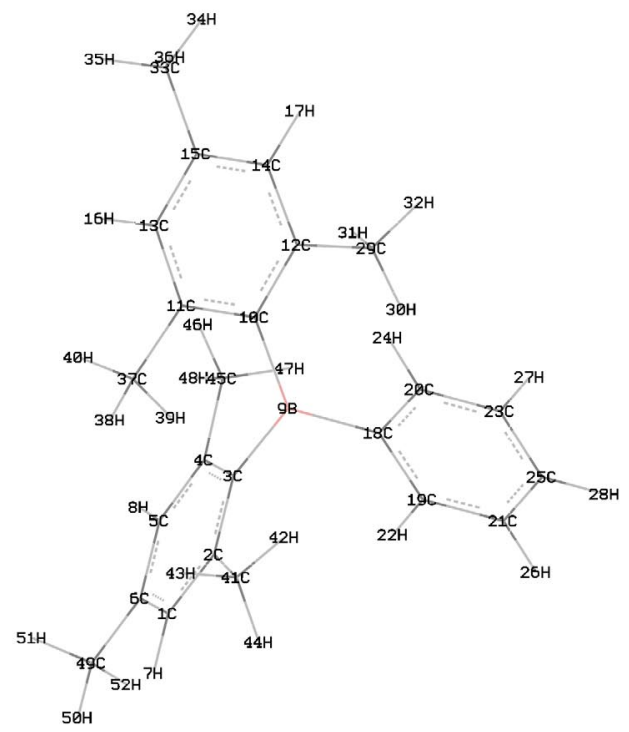

\begin{tabular}{|c|c|c|c|c|}
\hline$\#$ & Symbol & $X$ & $Y$ & $Z$ \\
\hline 1 & C & -3.622761 & -0.858060 & -0.842565 \\
\hline 2 & C & -2.387981 & -0.199872 & -0.942893 \\
\hline 3 & C & -1.389058 & -0.397247 & 0.044655 \\
\hline 4 & C & -1.691241 & -1.282461 & 1.120297 \\
\hline 5 & C & -2.937921 & -1.905602 & 1.191024 \\
\hline 6 & C & -3.923880 & -1.710486 & 0.216382 \\
\hline 7 & H & -4.366912 & -0.695420 & -1.621227 \\
\hline 8 & H & -3.147925 & -2.563653 & 2.033036 \\
\hline 9 & B & 0.000011 & 0.366361 & -0.000028 \\
\hline 10 & C & 1.389020 & -0.397355 & -0.044635 \\
\hline 11 & C & 1.691099 & -1.282807 & -1.120114 \\
\hline 12 & C & 2.388026 & -0.199819 & 0.942805 \\
\hline 13 & C & 2.937771 & -1.905968 & -1.190824 \\
\hline 14 & C & 3.622799 & -0.858021 & 0.842492 \\
\hline 15 & C & 3.923825 & -1.710650 & -0.216319 \\
\hline 16 & H & 3.147698 & -2.564193 & -2.032718 \\
\hline 17 & H & 4.367018 & -0.695227 & 1.621057 \\
\hline 18 & C & 0.000073 & 1.940457 & -0.000078 \\
\hline 19 & C & -1.107440 & 2.677660 & 0.474733 \\
\hline 20 & C & 1.107593 & 2.677629 & -0.474911 \\
\hline 21 & C & -1.106131 & 4.071659 & 0.488638 \\
\hline 22 & H & -1.978427 & 2.141310 & 0.841669 \\
\hline 23 & C & 1.106305 & 4.071626 & -0.488844 \\
\hline 24 & H & 1.978576 & 2.141264 & -0.841832 \\
\hline 25 & C & 0.000092 & 4.772029 & -0.000111 \\
\hline 26 & H & -1.966809 & 4.613386 & 0.872935 \\
\hline & & & & \\
\hline
\end{tabular}

\begin{tabular}{|l|l|l|l|l|}
\hline 27 & H & 1.966990 & 4.613332 & -0.873155 \\
\hline 28 & H & 0.000103 & 5.859331 & -0.000128 \\
\hline 29 & C & 2.165927 & 0.645067 & 2.185740 \\
\hline 30 & H & 1.314161 & 1.320998 & 2.095105 \\
\hline 31 & H & 1.988216 & 0.004779 & 3.060815 \\
\hline 32 & H & 3.051157 & 1.252154 & 2.409471 \\
\hline 33 & C & 5.266269 & -2.399835 & -0.319695 \\
\hline 34 & H & 5.884958 & -2.203437 & 0.562135 \\
\hline 35 & H & 5.152192 & -3.487064 & -0.414807 \\
\hline 36 & H & 5.824664 & -2.058406 & -1.201261 \\
\hline 37 & C & 0.701946 & -1.549246 & -2.239647 \\
\hline 38 & H & -0.240729 & -1.956549 & -1.860165 \\
\hline 39 & H & 0.456896 & -0.634957 & -2.794670 \\
\hline 40 & H & 1.114786 & -2.265139 & -2.957982 \\
\hline 41 & C & -2.165781 & 0.644826 & -2.185936 \\
\hline 42 & H & -1.313975 & 1.320713 & -2.095352 \\
\hline 43 & H & -1.988090 & 0.004422 & -3.060929 \\
\hline 44 & H & -3.050960 & 1.251953 & -2.409771 \\
\hline 45 & C & -0.702198 & -1.548607 & 2.239996 \\
\hline 46 & H & 0.240557 & -1.955902 & 1.860708 \\
\hline 47 & H & -0.457306 & -0.634181 & 2.794869 \\
\hline 48 & H & -1.115061 & -2.264395 & 2.958425 \\
\hline 49 & C & -5.266325 & -2.399669 & 0.319768 \\
\hline 50 & H & -5.884880 & -2.203568 & -0.562222 \\
\hline 51 & H & -5.152235 & -3.486863 & 0.415252 \\
\hline 52 & H & -5.824870 & -2.057973 & 1.201137 \\
\hline & & & & \\
\hline
\end{tabular}

